\title{
Influence of a pre-shot dynamic stretching routine on free throw performance
}

\author{
Igor Vučković ${ }^{1}$, Aleksandar Gadžić ${ }^{2}$, Vanja Sladojević ${ }^{1}$, Aleksandar Kukrić $^{1}$, Mladen Mikić ${ }^{3}$, \\ Marko Stojanović3
}

${ }^{1}$ Faculty of Physical Education and Sport, University of Banja Luka, Bosnia and Herzegovina; ${ }^{2}$ Faculty of Physical Education and Sports Management, Singidunum University, Belgrade, Serbia; ${ }^{3}$ Faculty of Sport and Physical Education, University of Novi Sad, Serbia

\section{Summary}

Study aim: The aim of the present study was to examine a single movement of dynamic stretching (SMDS) of the shooting arm as a pre-shot routine for free throw performance (FTP).

Material and methods: The sample consisted of 60 junior and senior basketball players from the youth league of Bosnia and Herzegovina $(\mathrm{B} \& \mathrm{H})$, and the national level - the First Division of B\&H. The authors found that some players during a game and training sessions apply an SMDS of the shooting arm as a pre-shot routine for FTP. Since previous literature suggests that length of the routine and pre-performance behaviors are quite different among players of all levels, the sample was divided based on the number of training hours per week (lower/higher number of training hours) and basketball experience (experienced/less experienced). The procedure involves every player performing five free throws (FTs) without prior stretching, five FTs immediately after SMDS of the triceps muscle and five FTs after SMDS of the m. flexor carpi radialis.

Results: After the first SMDS (m. triceps brachii) the percentage of FT slightly decreased in the first following attempt. After the second stretching (m. flexor carpi radialis), a significant FT percentage drop was noted in the first following FT for the whole sample. Players with more experience and more training hours per week had poorer results after the stretching. Conclusions: The SMDS routine did not enhance the FTP and it had a rather harmful effect on FTP, especially SMDS of the flexor carpi radialis muscle, and the authors do not recommend SMDS before the FTP.

\section{Key words: Triceps brachii - Flexor carpi radialis - Dynamic stretching}

\section{Introduction}

Basketball is one of the most dynamic and fastestgrowing sports today. That is the case for many reasons. One of them is that basketball is a very popular spectator sport, particularly on television. The international growth of basketball is so huge that there are over 200 countries that have basketball federations. Basketball as a sport keeps people of all ages and both sexes engaged and it is now played indoors and outdoors in all seasons [32]. The game of modern basketball involves a vast number of technical and tactical skills and players' ability to execute them in competitive situations directly affects the outcome of the game. Every basketball coach knows the importance of shooting as a fundamental technical skill in basketball. Free throw shooting, for many coaches, represents a decisive factor for their teams' success or failure in a basketball game [23]. This especially applies to so-called close games which are often decided by better free-throw performance (FTP). A previous study examined the differences between the performance of winning and losing basketball teams in the final tournament of the European club championships from 1992 to 2000 [34]. The authors found that free throw percentage, defensive rebounds, and field goal percentage were the critical factors that determined success. Another study focused on identification of critical performance indicators that most distinguish between winning and losing performances within basketball matches of the European Basketball Championship 2007 [10]. Researchers identified six critical performance indicators when only the close matches were considered. In those matches, the percentage of successful free throws $(\mathrm{p}<0.001)$ seemed to be the most crucial performance 
indicator that distinguished between winning and losing teams. The ability to successfully perform a free throw (FT) depends on a large number of parameters impacting the FT, and authors find that trajectory parameters can be correlated with success [29]. They identified five factors that a player should focus on in order to improve FT shooting: the height of release, the launch velocity, the vertical launch angle, the left-right deviation, and the backspin.

Beside the different trajectory parameters, or throwing mechanics, other authors claimed that success in FT is under the influence of: performance routine, relaxation, selfesteem, and concentration [19]. Numerous studies have focused on the relationships between psychological traits of basketball players and their percentage of successful free throws [12, 31, 37].

Previously it has been suggested that a method to develop a consistent set of cognitions and behaviors that are executed before performance of the motor skill might help athletes perform a skill successfully [6]. It is believed that pre-performance routines (PPR) can have a positive impact on performance in sport [25]. A PPR in sport has been an area of specific interest to sport psychologists, coaches, and performers over the past 30 years. Even though the use of PPR is more often implemented with closed skills (such as a golf shot, basketball free throw, long jump, hockey penalty flick, etc.), they have also been examined in some open skill sports [7]. The PPR research in sport to date is abundant and apart from basketball includes football [16], gymnastics [5], diving [17], volleyball [35], tennis [24], track and field athletics [8], and many more. However, empirical evidence regarding the effectiveness of these routines has not been universally supported [18]. Lonsdale and Tam [20] investigated the effects of duration and specific pattern of behaviors exhibited before each FT during 14 NBA play-off games. They did not find a difference in the success rates of shots associated with brief, long, and regular duration routines. However, they found that players were more successful when they followed their dominant behavioral sequence $(83.77 \%$ success $)$ than when they deviated from their specific behavioral pattern. The results of a recent study with high school basketball players suggest that players who applied a PPR outperformed those who did not use a pre-performance routine, although with very small differences [30].

Czech, Ploszay, and Burke [11] found that athletes who performed and maintained the same pre-shot routine had a higher FT percentage than those who did not maintain a PPR or ritual.

While working with basketball players of different age and experience in Bosnia and Herzegovina, the authors noticed that some players during a game and training sessions apply a single movement of dynamic stretching of the shooting arm as a PPR for free throw performance (FTP). In previous study researchers found that static stretching of the shooting arm did not improve performance of FT [36]. Based on previous literature, it appears that length of the routine and pre-performance behaviors are quite different among players of all levels; therefore, in the present study, an attempt was made to examine dynamic stretching of the shooting arm as a pre-shot routine for FTP.

From the anatomical point of view, the muscles that lift and throw the ball to the hoop are: triceps brachii, deltoideus, flexor carpi radialis and biceps brachii. The movement contribution of these muscles was established by electromyography of FTP [39]. A number of studies have shown reduced sports performance immediately after stretching exercise $[9,20,22,33]$. That is the main reason we assumed that a single movement of dynamic stretching (SMDS) would not improve the FTP.

\section{Material and methods}

The sample of 60 participants was selected from junior and senior basketball players, mean age $19.55 \pm 4.35$. There were 24 junior players (age ranged from 16 to 17 years; mean $16.63 \pm 0.49$ years) who compete in the youth league of Bosnia and Herzegovina (B\&H), and 36 senior players ranging from 18 to 37 years of age $(21.5 \pm 4.68 ; 12$ compete at national level - First Division of B\&H, 24 compete in Second Division of B\&H). Based on the number of training hours per week $(8.05 \pm 2.24$ hours/week), the participants were divided into a group with 8.05 or more hours per week $(n=31 ; 9.97 \pm 1.43$ hours/week) and up to 8.05 hours $(\mathrm{n}=29 ; 6.00 \pm 0.00$ hours/week) groups. We analyzed the significance of differences between these two groups and its potential influence on FT accuracy. For observation of the potential influence of the overall basketball experience on FT accuracy, for the purpose of the analysis, based on mean experience ( $9.2 \pm 4.59$ years), we divided the sample into groups of over 9.2 years $(\mathrm{n}=24 ; 13.67 \pm 3.46$ years $)$ and below 9.2 years ( $\mathrm{n}=36 ; 6.22 \pm 2.22$ years) of experience. All procedures were conducted in accordance with the ethical standards of the Helsinki Declaration and all the participants gave their written informed consent.

Although a basketball player rarely has the opportunity to perform five consecutive FTs in game situations (usually it is one, two or three free throws), we decided to include five FTs in order to follow a trend of success through elapsed time ( $\sim 25^{\prime}$ after stretching). In order to gain full insight into FTP we wanted to examine three situations: 1) without stretching, 2) after the SMDS of muscle triceps brachii and 3) after the SMDS of flexor carpi radialis. We presumed proper observation of the trend of successful FTP would be provided with five FTs in all of the three series of throws. The immediate effects of SMDS were expected in the sixth and eleventh FT (the first ones after the first and the second stretching procedures). 
The previous warm-up was eliminated due to a possible unequal influence on the participants and their accuracy especially if arm stretching has been done. As mentioned above, players usually perform up to three consecutive FTs in the game situation and on top of that we added two FTs in the protocol to equalize the starting positions in all three situations, and thus we proposed 15 FTs in total.

Without a prior warm-up, the basketball player stands on the FT line, takes the ball, and performs two practice FTs that do not count in the score. It was not allowed to tap the ball between the attempts, but a correction in the arm was permitted. After that, the player performs five FTs. The score taker writes down the score. Then, the player performs an SMDS of the triceps brachii muscle of the shooting arm (Fig. 1). The following sequence is that he executes five FTs, which count in the score. Furthermore, at the time keeper's signal he starts to perform an SMDS of forearm muscles (flexor carpi radialis) (Fig. 2). After that, he performs five FTs that count in the score too. Applied intensity of stretching was submaximal as recommended by Chaouachi et al. [4].

All of the tests were conducted in a sports hall, during the competitive season, before the usual training session. FTs were performed with a Spalding TF500 size 7 basketball.

\section{Results}

Percentages of successful FTs were calculated after performing the three described procedures and trends of success were analyzed. We performed the $\chi^{2}$ test to determine differences between arithmetic means of the $5^{\text {th }}$ and $6^{\text {th }}$ series of FTs, and $10^{\text {th }}$ and $11^{\text {th }}$ series respectively. The Mann-Whitney U test was applied to determine differences in successful FTs between the experienced and less experienced players, and between the players with more training hours and fewer training hours per week. Microsoft Office Excel (Version 2007; Microsoft, Redmond, WA, USA) and SPSS (Version 20; SPSS, IBM Corp., Armonk, NY) were used to process the data.

The results of the current study were presented in five figures. The first five columns in figures (red color) represent percentages of successful FTs before the dynamic stretching. The second five columns in figures (yellow color) show the percentage of successful FTs after an SMDS of the triceps brachii muscle (upper arm). The third set of columns in figures (blue color) show the percentage of successful FTs after the SMDS of the flexor carpi radialis muscle (forearm). We tried to follow the trend of successful FTP from the first to the fifth throw in all three series of throws.

As presented in Figure 3, from the first to the fifth FT success showed a stable trend, except for the second FT. It seems that after the second FT the feedback stabilized the process of execution of the complex movement. Since a free throw lasts longer than a third of a second, feedback can help in control, and accuracy of movement performance (closed control loop). The speed of information processing improves with the number of repetitions. In the background of that is partial transfer from closed loop control to open loop control, and motor programs are used for execution of the movement [1]. After the SMDS of the triceps brachii muscle, the percentage of successful FTs slightly decreases

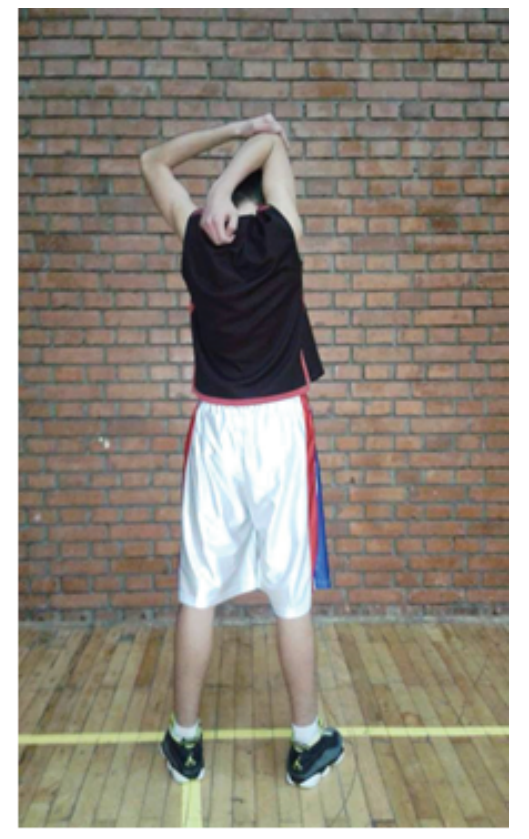

Fig. 1. Dynamic stretching of triceps brachii

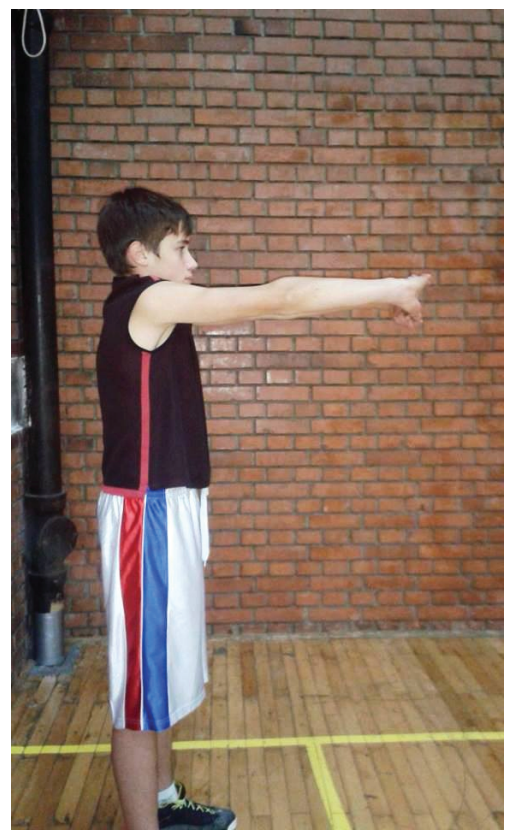

Fig. 2. Dynamic stretching of flexor carpi radialis 


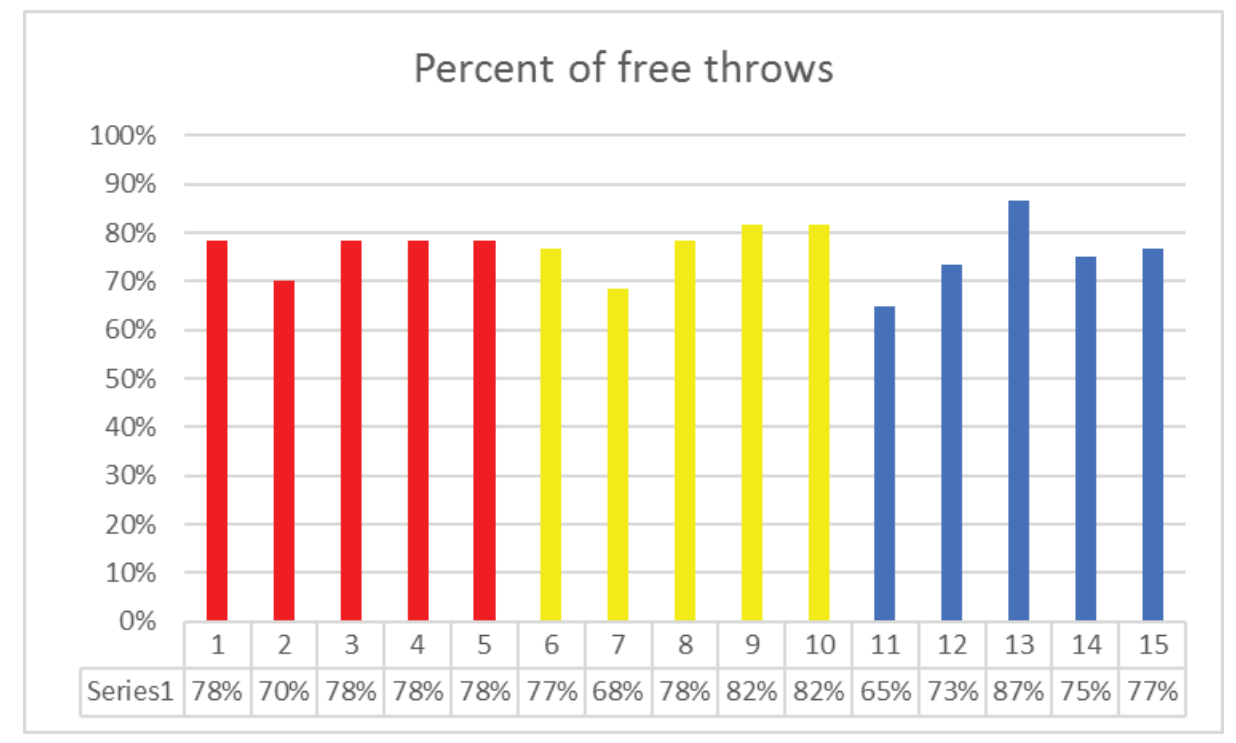

Fig. 3. Percentage of free throws before stretching, after stretching of triceps brachii muscle and after stretching of flexor carpi radialis for the whole sample

in the first following attempt (sixth free throw). After the second stretching procedure (SMDS of the flexor carpi radialis muscle), a significant FT percentage drop was noted in the first shot ( $11^{\text {th }}$ column in Figure 3$)$ after the stretching procedure, from $82 \%$ to $65 \% ; \chi^{2}=4.261 ; \mathrm{df}=1 ; p=0.039$. The first five FTs' success rate was $76.4 \%$, that of the second five throws was $77.4 \%$, and that of the third five throws was $75.4 \%$. Apparently, the first SMDS procedure did not cause a significant drop in FTP, but the second one did and it implies that further SMDS had a stronger and longer detrimental effect on FTP, since the last five FTs showed considerable fluctuations.

Figure 4 shows that FTP of basketball players with fewer training hours per week has rather an inconsistent trend in all three scenarios. A possible explanation might simply be the fact that they practice less and the process of organization and execution of a precise movement is not as effective as it needs to be. Furthermore, the basketball players with fewer training hours per week have a lower percentage of successful FTs overall compared to basketball players with more training hours per week $(72 \%$ to $80.4 \%$ ), which is quite logical.

Analyses of the first FTs after the SMDS of triceps and flexor carpi radialis (columns 6 and 11 in Figure 5) shows that basketball players with more training hours per week were more susceptible to immediate negative effects of SMDS of aforementioned muscles. The first series of FT demonstrated a high FT percentage (83.2\%)

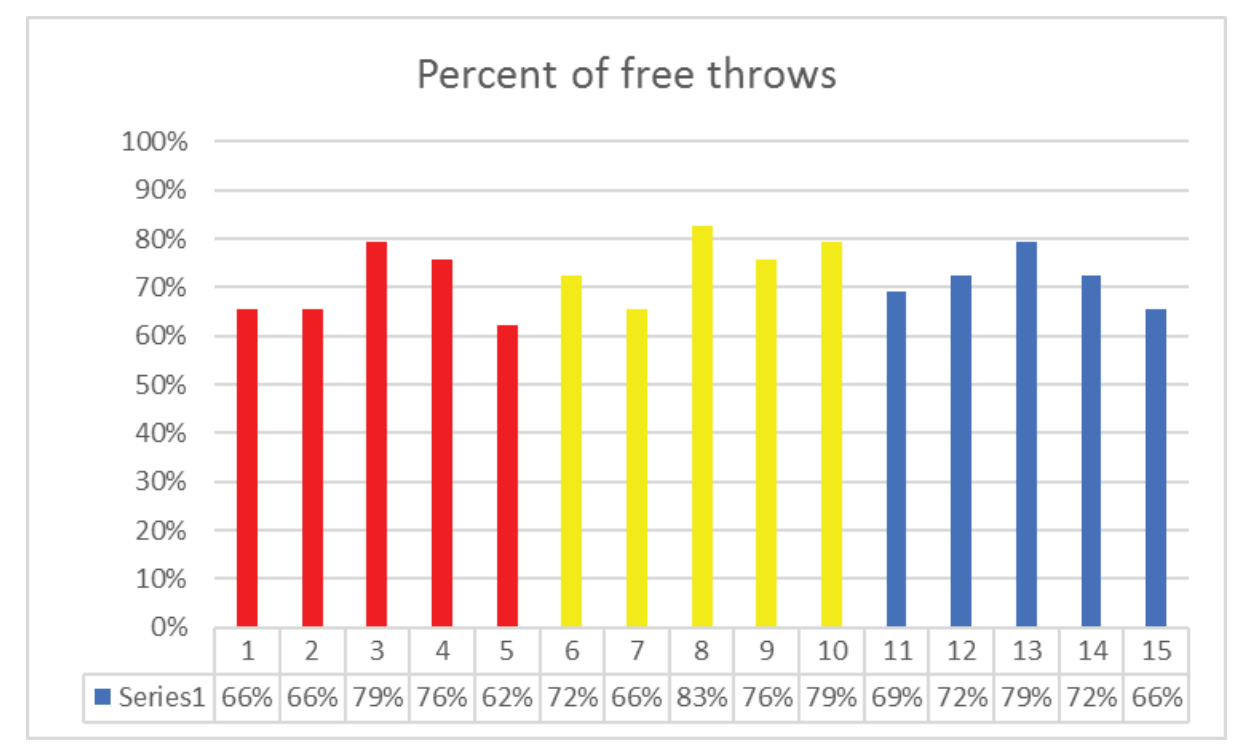

Fig. 4. Percentage of free throws before stretching, after stretching of triceps brachii muscle and after stretching of flexor carpi radialis for group of players with fewer training hours per week $(\mathrm{n}=29)$ 


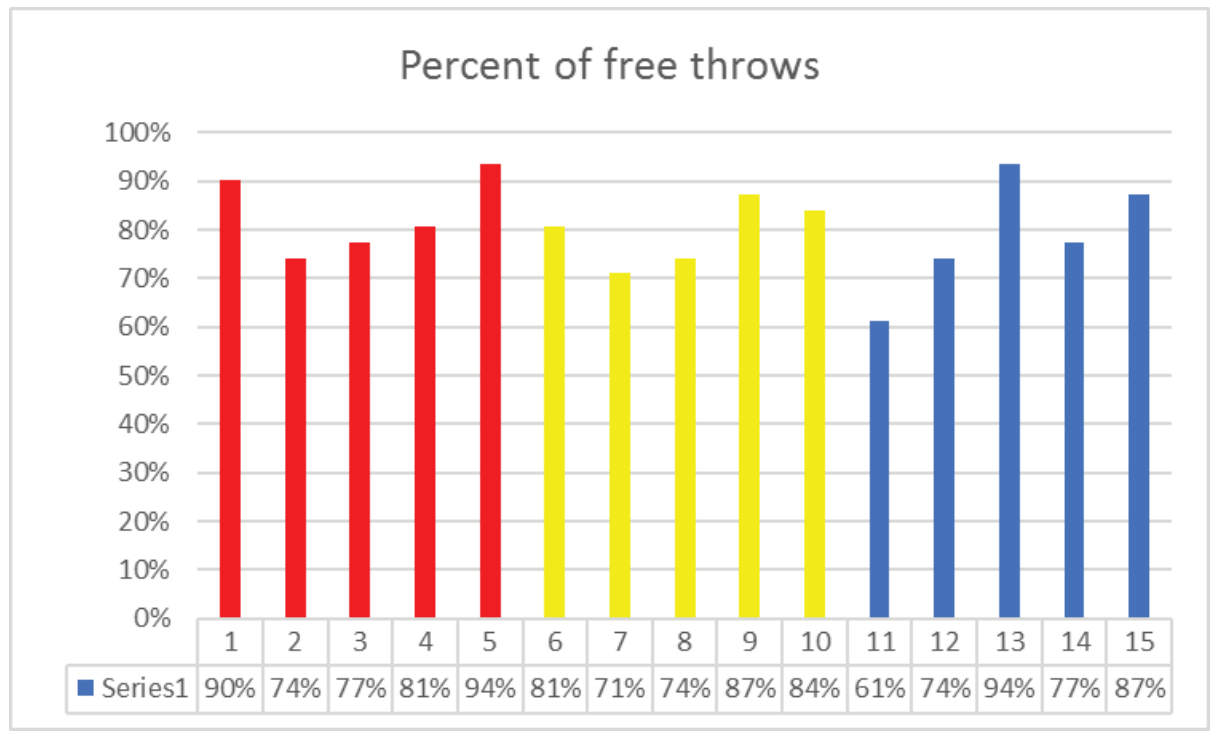

Fig. 5. Percentage of free throws before stretching, after stretching of triceps brachii muscle and after stretching of flexor carpi radialis for group of players with more training hours per week $(n=31)$

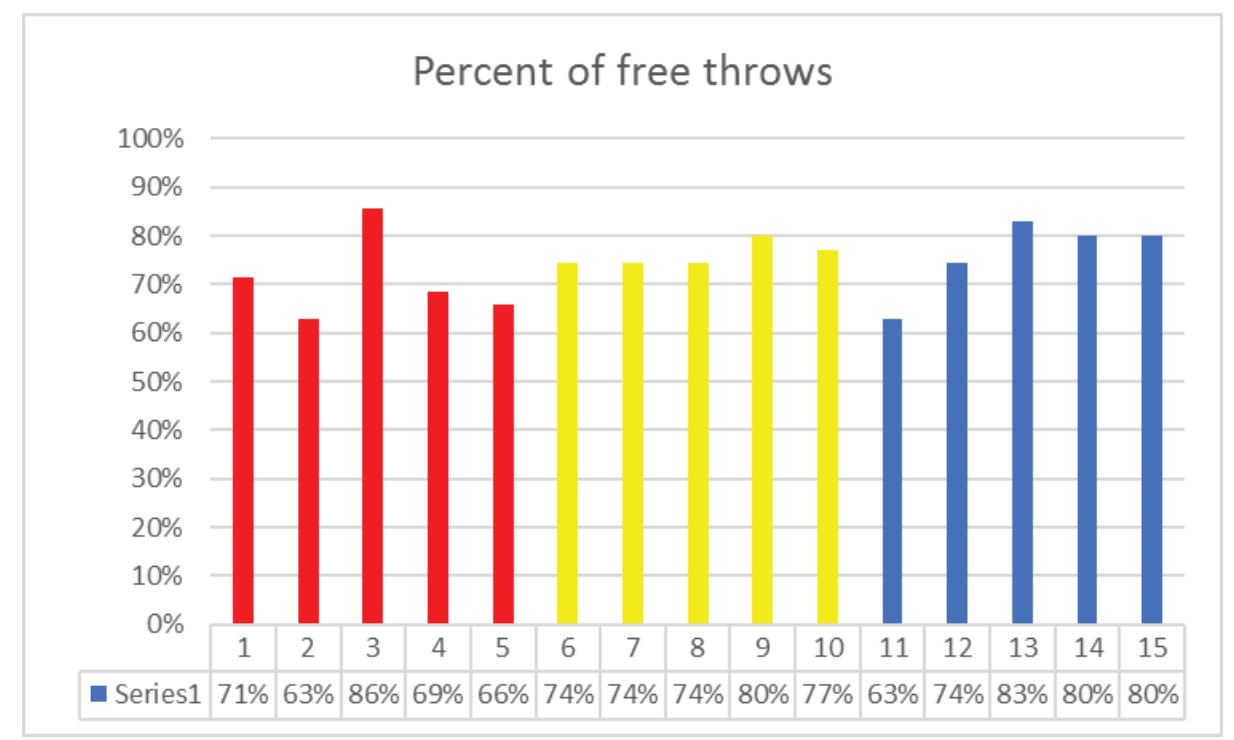

Fig. 6. Percentage of free throws before stretching, after stretching of triceps brachii muscle and after stretching of flexor carpi radialis for less experienced group of players $(n=35)$

but the second (79.4\%) and the third series (78.6\%) clearly indicated a decreasing trend of FTP. This finding indicates that players with more training hours per week may be followers of different PPR and therefore the SMDS routine caused more distractions to their usual routine.

FTP of the less experienced group of players (Fig. 6), after the first series of FTs, demonstrated a somewhat surprising increase in FT percentage in the following second and third series. A plausible explanation for this finding might be that less experienced players probably fall into the group of junior players of this study (mean age $16.63 \pm 0.49$ years) and they did not develop stable and consistent PPRs; hence the applied SMDS had less impact on their FTP.

Considering all $15 \mathrm{FTs}$, the less experienced group of basketball players had a lower percentage than the experienced group of basketball players ( $74.3 \%$ to $79.5 \%)$, which is similar to previous analyses of players with lower and higher numbers of training hours per week. Contrary to our expectations, both stretching procedures had a more negative impact on the shooting accuracy of experienced players than among less experienced ones and the reason for that could be in fact that we did not know normal PPRs of any group of players, but it is plausible that experienced players have adopted more effective PPRs than SMDS. 


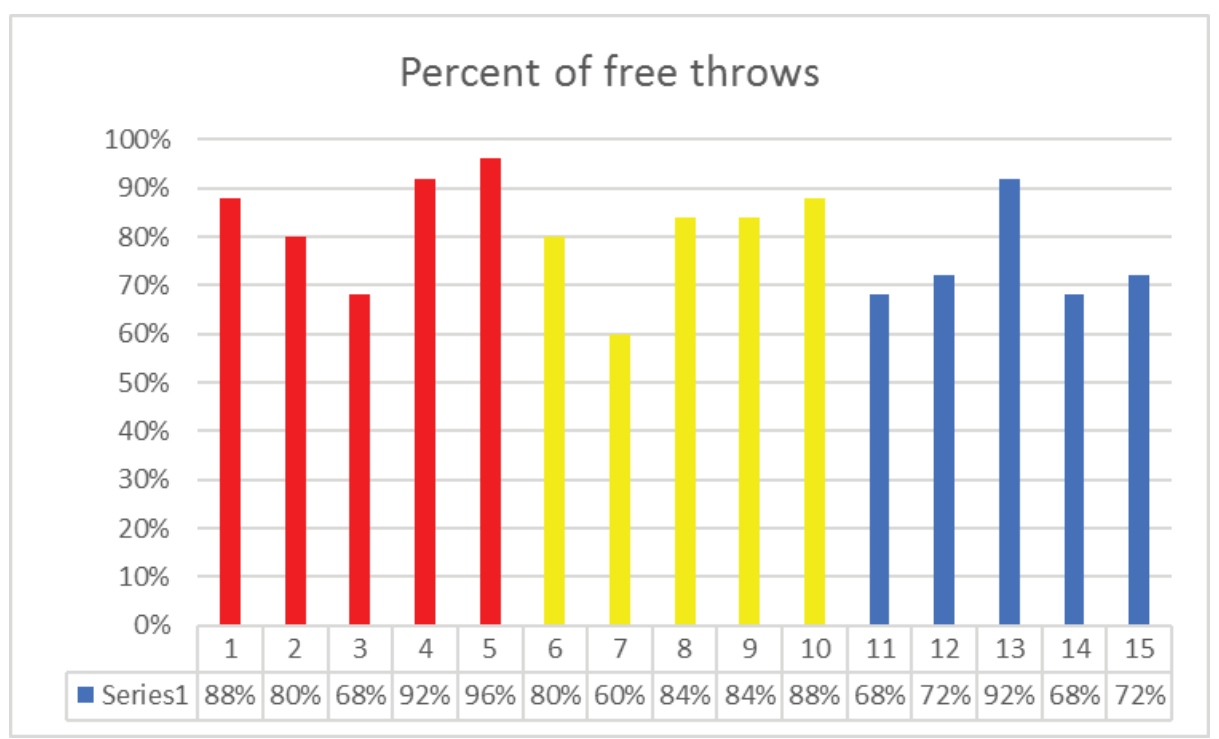

Fig. 7. Percentage of free throws before stretching, after stretching of triceps brachii muscle and after stretching of flexor carpi radialis for experienced group of players $(n=25)$

\section{Discussion}

The aim of this study was to examine how the pre-shot routine of an SMDS impacts FTP. Our initial assumption was that both stretching procedures, of upper arm and forearm muscles, would negatively affect the FTP. Results from the whole sample indicate two key findings. First, SMDS of the triceps muscle caused just a minor drop in FT percentage (Figure 3; columns 5 and 6). Second, there was a significant drop in FT percentage in the first FT after the stretching of the flexor carpi radialis muscle $\left(11^{\text {th }}\right.$ column, drop of $17 \%$ ). It seems that SMDS of the flexor carpi radialis muscle had a negative impact on FTP. The percentage of the following two FTs (columns 12 and 13) has increased; considering the widely documented nonexistence of long-lasting effects of stretching exercises on the resistance of the muscle-tendon unit $[31,32,33]$, we assumed that negative effects of the SMDS of the flexor carpi radialis muscle have a relatively short duration $\left(\sim 5^{\prime}-10^{\prime}\right)$.

Considering the amount of practice among the group of players, the Mann-Whitney $U$ test showed significant differences in the first $(p=0.021)$ and the fifth $(p=0.003)$ series of FTs in favor of players with more training hours per week. These findings are quite logical and the overall percentage of successful FTs is better for basketball players with more training hours per week ( $72 \%$ to $80.4 \%$ ). Conversely, basketball players with more training hours per week contributed more to the FT percentage drop after the fifth and sixth series of throws in the whole sample. That is more evident after the SMDS of the flexor carpi radialis. A possible explanation might lie in one of the limitations of the current study since the authors did not identify the players who regularly perform dynamic stretching as a PPR. It might be the case that players with more training hours per week do not use this practice at all and consequently they might be more distracted. Both groups gradually improved the $5^{\text {th }}$ and $11^{\text {th }} \mathrm{FT}$, confirming that the speed of information processing improves with the number of repetitions [1].

Regarding players' experience, where players were grouped into less experienced and experienced groups, the Mann-Whitney U test showed significant differences between the groups only in the fourth $(p=0.031)$ and fifth $(p=0.005)$ series of FTs in favor of experienced players. Once again, these results seem logical and the experienced group of basketball players recorded a better percentage of successful FTs than less experienced players ( $74.3 \%$ to $79.5 \%$ ). Experienced players had more fluctuations in FT accuracy and their percentage drop after SMDS of the triceps muscle was more notable (from $88 \%$ to $68 \%$ ) than in less experienced players (from $77 \%$ to $63 \%$ ). The authors of the present study noticed that the SMDS routine was more common in junior players. It is plausible that senior players, who have well established other PPRs for a longer period of time, were more perturbed with the change of their "ritual" that facilitates performance and gives performers control over their motor, emotional, and cognitive behaviors.

Research indicates that stretching does not affect accuracy-related movements such as FTP [21]. Conversely, in a previous study, the authors found negative effects of static stretching on FTP [36]. Static stretching in that study was performed for 15 seconds on the triceps muscle (drop in FT percentage of $11 \%$ ) and flexor carpi radialis muscle (drop in FT percentage of 8\%). Although the drop in FT percentage was not statistically significant, the authors do 
not recommend static stretching routines before the execution of FTs. Unlike that study, where static stretching PPR was applied, the current study did not find a negative influence of SMDS of the triceps muscle. However, the negative influence of SMDS of the flexor carpi radialis muscle was statistically significant. Dynamic stretching is usually considered preferable to static stretching in the preparation for physical activity, but a systematic review of the literature has shown that there is no overall evidence for substantial performance enhancements after dynamic stretching [2].

Basketball FT shooting is an unnatural movement that has to be learned and is characterized by individual shooting patterns; as such, a typical textbook technique which every athlete should aim for cannot be concluded. Research confirmed an overall increase of intra-individual movement pattern stability of shooting in basketball with increasing skill level, and distractions of movement patterns, such as dynamic stretching, probably affect the performance [31].

\section{Conclusion}

In sports that have a number of self-paced motor skills, almost all athletes, in an attempt to maximize performance, have established PPRs. One of them is basketball with the FT procedure. Over the years, we have seen all sorts of routines from the foul line in basketball.

Some previous experimental studies have examined performance outcomes using two conditions, with and without the use of a PPR [13, 26, 32]. The general conclusion was that performance is significantly better when using a PPR, but researchers have not examined the effect of substituting one behavioral routine for another. However, a more recent study confirmed that players were more successful when they followed their dominant behavioral sequence $(83.77 \%$ success $)$ than when they deviated from their specific behavioral pattern (71.43\% success) [27].

In the current study, we investigated the influence of pre-shot SMDS of the shooting arm in players with different levels of experience and training hours per week. Our findings clearly suggest that SMDS routines did not enhance the FTP. On the contrary, they had a rather harmful effect on FTP, especially dynamic stretching of the flexor carpi radialis muscle. Therefore the authors do not recommend dynamic stretching before the FTP.

Future research should focus on broadening the subject base and varying the conditions in terms of physically performing or not performing PPRs in order to be more insightful. Future studies could examine the effects of physically performing the routine and without performing the routine to see whether there is a decrease in accuracy without routine performance. Furthermore, subjects with few training hours per week and less experienced ones should not be primarily targeted since their shooting trend after the stretching routines seems quite unstable.

\section{Conflict of interest: Authors state no conflict of interest.}

\section{References}

1. Abernethy B., Kippers V., Hanrahan S.J. Pandy M., MacManus A., Mackinnon L. (2012) Biophysical foundations of human movement 3rd ed. Human Kinetics, Champaign, IL.

2. Behm D.G., Blazevich A.J., Kay A.D., McHugh M. (2016) Acute effects of muscle stretching on physical performance, range of motion, and injury incidence in healthy active individuals: a systematic review. Appl. Physiol. Nutr. Metab., 41: 1-11.

3. Björklund M., Hamberg J., Crenshaw A.G. (2001) Sensory adaptation after a 2-week stretching regimen of the rectus femoris muscle. Arch. Phys. Med. Rehabil., 82: 1245-1250.

4. Chaouachi A., Castagna C., Chtara M., Brughelli M., Turki O., Galy O., Chamari K., Behm D.G. (2010) Effect of warm-ups involving static or dynamic stretching on agility, sprinting, and jumping performance in trained individuals. J. Strength Cond. Res., 24: 2001-2011.

5. Clowes H., Knowles Z. (2013) Exploring the effectiveness of pre-performance routines in elite artistic gymnasts: a mixed method investigation. Sci. Gymnast. J., 5(2): 27-40.

6. Cohn P.J. (1990) Pre-performance routines in sport: Theoretical support and practical applications. Sport Psychol., 4: 301-312.

7. Cotterill S. (2010) Pre-performance routines in sport: current understanding and future directions. Int. Rev. Sport Exerc. Psychol., 3(2): 132-153.

8. Cotterill S., Greenlees I. (2003) Temporal consistency of pre-performance routines in world-class polevaulters. Proceedings from the VIIth IOC World Congress on Sport Sciences, Athens. Athens: Organising Committee for the Olympic Games.

9. Cramer J.T., Housh T.J., Johnson G.O., Miller J.M., Coburn J.W., Beck T.W. (2004) Acute effects of static stretching on peak torque in women. J. Strength Cond. Res., 18: 236-241.

10. Csataljay G., O’Donoghue P., Hughes M., Dancs H. (2009) Performance indicators that distinguish winning and losing teams in basketball. Int. J. Perf. Anal. Sport, 9: 60-66.

11. Czech D.R., Ploszay A., Burke K.L. (2004) An examination of the maintenance of preshot routines in basketball free throw shooting. J. Sport Behav., 27: 323-329.

12. Foster D., Weigand, D., Baines D. (2006) The Effect of Removing Superstitious Behavior and Introducing a Pre- 
Performance Routine on Basketball Free-Throw Performance. J. Appl. Sport Psychol., 18: 167-171.

13. Gayton W.F., Cielinski K.L., Francis-Keniston W.J., Hearns J.F. (1989) Effects of preshot routine on freethrow shooting. Percept. Mot. Skills, 68: 317-318.

14. Halbertsma J.P., Mulder I., Göeken L.N., Eisma W.H. (1999) Repeated passive stretching: Acute effect on the passive muscle moment and extensibility of short hamstrings. Arch. Phys. Med. Rehabil., 80: 407-414.

15. Harvey L.A., Byak A.J., Ostrovskaya M. Glinsky J., Katte L., Herbert R.D. (2003) Randomised trial of the effects of four weeks of daily stretch on extensibility of hamstring muscles in people with spinal cord injuries. Aust. J. Physiother, 49: 176-181.

16. Hazell J., Cotterill S., Hill D.M. (2014) An exploration of pre-performance routines, self-efficacy, anxiety and performance in semi-professional soccer. Eur. J. Sport Sci., 14(6): 603-610.

17. Highlen P.S., Bennett B.B. (1983) Elite divers and wrestlers: A comparison between open and closed skill athletes. J. Sport Exerc. Psychol., 5: 390-409.

18. Jackson R.C. (2003) Pre-performance routine consistency: Temporal analysis of goal kicking in the Rugby Union World Cup. J. Sport Sci., 21: 803-814.

19. Karalejić M., Jakovljević S. (2001) Osnove košarke. Beograd: Fakultet sporta i fizičkog vaspitanja.

20. Kay A.D., Blazevich A.J. (2008) Reductions in active plantar flexor moment are significantly correlated with static stretch duration. Eur. J. Sport Sci., 8: 41-46.

21. Knudson D., Mache M., Kotte J. (2004) Stretching Has No Effect on Free Throw Shooting Accuracy. Med. Sci. Sports Exerc., 36: 206.

22. Knudson D., Noffal G. (2005) Time course of stretching induced isometric strength deficits. Eur. J. Appl. Physiol., 94: 348-351.

23. Kozar B., Vaughn R.E., Whitfield K.E., Lord R.H., Dye B. (1994) Importance of free-throws at various Stages of basketball games. Percept. Mot. Skills, 78: 243-248.

24. Lautenbach F., Laborde S., Mesagno C., Lobinger B.H., Achtzehn S., Arimond F. (2014) Nonautomated PrePerformance Routine in Tennis: An Intervention Study J. Appl. Sport Psychol., 27(2): 123-131.

25. Lidor R., Singer R.N. (2000) Teaching pre-performance routines to beginners. J. Phys. Edu. Recreat Dance, 71: 34-36.

26. Lobmeyer D.L., Wasserman E.A. (1986) Preliminaries to free throw shooting: superstitious behavior? J. Sport Behav., 9: 70-78.

27. Lonsdale C., Tam J.T.M. (2008) On the temporal and behavioural consistency of pre-performance routines: An intra-individual analysis of elite basketball players' free throw shooting accuracy, J. Sport Sci., 26: 259-266.

28. Magnusson S.P., Aagard P., Simonsen E. BojsenMoeller F. (1998) A biomechanical evaluation of cyclic and static stretch in human skeletal muscle. Int. J. Sports Med., 19: 310-316.

29. Maymin A., Maymin P., Shen E. (2012) The Individual Factors of Successful Free Throw Shooting. J. Quant. Anal. Sports, 8: Published online 2012-10-09. DOI: 10.1515/1559-0410.1414.

30. Phelps A., Kulinna P. (2015) Pre-performance routines followed by free throw shooting accuracy in secondary basketball players. Biomed. Hum. Kinetics., 7: 171-176. DOI: 10.1515/bhk-2015-0025.

31. Schmidt A. (2012) Movement pattern recognition in basketball free-throw shooting. Hum. Mov. Sci., 31: 360-382.

32. Southard D., Miracle A., Landwer G. (1989) Ritual and free-throw shooting in basketball. J. Sport Sci., 7: 163-173.

33. Stojanović M.D., Stojanović M.V., Veljović D., Međedović B., Ostojić S.M. (2009) Uloga i značaj istezanja u sportu. TIMS Acta, 3: 66-72.

34. Trninic S., Dizdar D., Luksic E. (2002) Differences between winning and defeated top quality basketball teams in final of European club championship. Coll. Antropol., 26: 521-531.

35. Velentzas K., Heinen T., Shack T. (2010) Routine Integration Strategies and their Effects on Volleyball Serve Performance and Players' Movement Mental Representation. J. Appl. Sport Psychol., 23(2): 209-222.

36. Vučković I., Gadžić A. (2016) Acute effects of static stretching of upper arm and forearm on the accuracy of free throws in basketball. Acta Gymn., 46: 122-128.

37. Wilson M., Vine S., Wood G. (2009) The influence of anxiety on visual attentional control in basketball free throw shooting. J. Sport Exerc. Psychol., 31: 152-168.

38. Wissel H. (2012) Basketball Steps to Success. Human Kinetics, Champaign, IL.

39. Zachry T., Wulf G., Mercer J., Bezodis N. (2005) Increased movement accuracy and reduced EMG activity as the result of adopting an external focus of attention. Brain Res. Bull., 67: 304-309.

\section{Received 23.08.2019 \\ Accepted 16.11.2019}

(c) University of Physical Education, Warsaw, Poland 